\title{
The knockdown of SNHG3 inhibits the progression of laryngeal squamous cell carcinoma by miR-340-5p/YAP1 axis and Wnt/ $\beta$-catenin pathway
}

\author{
R. KANG ${ }^{1, *}$, D. F. YAO ${ }^{2, *}$, G. Z. XU ${ }^{3}$, Y. H. ZHOU ${ }^{4, *}$ \\ ${ }^{1}$ Department of Otolaryngology, The Zhoukou Central Hospital, Zhoukou, Henan China; ${ }^{2}$ Department of Otolaryngology and Head and Neck \\ Surgery, The First Affiliated Hospital of Guangxi Medical University, Nanning, Guangxi, China; ${ }^{3}$ Department of Otolaryngology, Shang Cai Xie \\ He Hospital, Shangcai County, Zhumadian, Henan, China; ${ }^{4}$ Department of Otolaryngology, The First Affiliated Hospital of Henan University of \\ Science and Technology, Luoyang, Henan, China
}

*Correspondence: gytlor@163.com

${ }^{*}$ Contributed equally to this work.

Received October 22, 2019 / Accepted January 22, 2020

\begin{abstract}
Laryngeal squamous cell carcinoma (LSCC) is a common malignancy of the head and neck. Long noncoding RNAs (lncRNAs) play essential roles in the development and treatment of LSCC. However, the role and regulatory mechanism of lncRNA small nucleolar RNA host gene 3 (SNHG3) in LSCC progression remain largely unknown. Twenty-five paired LSCC tissues and normal samples were collected. The expression levels of SNHG3, Yes-associated protein 1 (YAP1), and microRNA-340-5p (miR-340-5p) were measured via quantitative real-time polymerase chain reaction or western blot. Cell viability, apoptosis, and glycolysis were investigated by 3-(4,5-dimethylthiazol-2-yl)-2,5-diphenyl-tetrazolium bromide, flow cytometry, and specific assay kit, respectively. The association between SNHG3 and miR-340-5p or miR-340-5p and YAP1 was assessed by dual-luciferase reporter assay. The expression of a protein involved in the Wnt/ $\beta$-catenin pathway was detected by western blot. The xenograft model was established to assess the anti-cancer role of SNHG3 inhibition in vivo. We found that the levels of SNHG3 and YAP1 were increased but the miR-340-5p expression was decreased in LSCC tissues and cells. The knockdown of SNHG3 or YAP1 inhibited cell viability and glycolysis but induced apoptosis in LSCC cells. Overexpression of YAP1 reversed the effect of SNHG3 knockdown on LSCC progression. SNHG3 could regulate YAP1 expression by competitively binding with miR-340-5p. Overexpression of miR-340-5p suppressed cell viability and glycolysis but promoted apoptosis in LSCC cells. Knockdown of SNHG3 repressed Wnt/ $\beta$-catenin pathway by regulating miR-340-5p and YAP1. The silencing of SNHG3 reduced LSCC xenograft tumor growth. In conclusion, knockdown of SNHG3 inhibited LSCC progression via inactivating Wnt/ $\beta$-catenin pathway by regulating the miR-340-5p/YAP1 axis.
\end{abstract}

Key words: laryngeal squamous cell carcinoma, SNHG3, miR-340-5p, YAP1, Wnt/ $\beta$-catenin pathway

Laryngeal cancer is a major malignancy of head and neck [1] and laryngeal squamous cell carcinoma (LSCC) comprises approximately $90 \%$ cases of laryngeal cancer [2]. Although the treatment of LSCC has gained great improvements, it remains a challenging clinical problem [3]. Glycolysis is a key process for meeting the metabolic requirement of cell survival [4]. Hence, understanding the regulatory mechanism of glycolysis in LSCC might be helpful for finding out new therapeutic strategies.

The long noncoding RNAs (lncRNAs) could function as sponges of microRNAs (miRNAs) by inhibiting miRNAmediated function to take part in the development and treatment of human diseases [5]. LncRNAs play essential roles in the prognosis, diagnosis, and therapy of laryngeal cancer, including LSCC [6]. The lncRNA small nucleolar
RNA host gene 3 (SNHG3) has been reported to function as an oncogenic lncRNA participating in proliferation and metastasis of hepatocellular carcinoma, breast cancer, and osteosarcoma [7-9]. Furthermore, a previous study suggests that SNHG3 is related to glycolysis of ovarian cancer [10]. An emerging report demonstrates that SNHG3 promotes cell proliferation and migration in laryngeal cancer [11]. Nevertheless, the role of SNHG3 in glycolysis and its potential mechanism in LSCC progression remain largely unknown.

miR-340-5p usually acts as a tumor-suppressive miRNA by inhibiting tumor development in cancers, such as lung cancer, glioblastoma, and osteosarcoma [12-14]. Moreover, increasing evidence uncovers that miR-340-5p is associated with the regulation of glycolysis in multiple cancers $[15,16]$. Importantly, the former finding suggests that miR-340-5p 
represses cell proliferation, migration as well as invasion in LSCC [17]. However, whether miR-340-5p is critical for the regulatory mechanism of SNHG3 is unexplored.

Yes-associated protein 1 (YAP1) is implicated in metabolism regulation, such as promoting glycolysis in human cancers [18]. Previous studies suggest that elevated YAP1 indicates poor prognosis of LSCC patients and contributes glycolysis and drug resistance in LSCC cells $[19,20]$. Wnt $/ \beta$ catenin signaling is a crucial downstream pathway of YAP1 and participates in cell metabolism [21,22]. The former work indicates that the $\mathrm{Wnt} / \beta$-catenin pathway is positively associated with cell proliferation, migration, invasion, and glycolysis in LSCC [23]. However, whether SNHG3 could regulate YAP1-mediated Wnt/ $\beta$-catenin signaling in LSCC is undetermined.

In the present research, we investigated the role of SNHG3 in LSCC progression. Moreover, we assessed the regulatory network of SNHG3 and miR-340-5p/YAP1/Wnt/ $\beta$-catenin. This study may provide a novel mechanism for understanding the pathogenesis of LSCC.

\section{Patients and methods}

Patients and clinical tissues. Twenty-five LSCC patients (20 males and 5 females; age range: $35-54$ years) were recruited from the Zhoukou Central Hospital. The cancer tissues and corresponding normal samples were harvested and stored at $-80^{\circ} \mathrm{C}$. All subjects have provided the written informed content and this study has got the approval of the Ethics Committee of the Zhoukou Central Hospital.

Cell culture. Human nasopharyngeal epithelial cells (NP69) and LSCC cell lines (TU177 and AMC-HN-8) were obtained from BeNa Culture Collection (Beijing, China). Cells were maintained in Roswell Park Memorial Institute (RPMI)-1640 medium (Thermo Fisher, Wilmington, DE, USA) containing 10\% fetal bovine serum (Zhejiang Tianhang Biotechnology, Huzhou, China) and $1 \%$ penicillin-streptomycin solution (Beyotime, Shanghai, China) in an incubator $\left(37^{\circ} \mathrm{C}\right.$ and $\left.5 \% \mathrm{CO}_{2}\right)$.

Quantitative real-time polymerase chain reaction (qRT-PCR). Total RNA was isolated via Trizol (Solarbio, Beijing, China). The complementary DNA (cDNA) was prepared using $500 \mathrm{ng}$ RNA and the TaqMan cDNA synthesis kit (Thermo Fisher). After dilution of 1:5, the synthesized cDNA was mixed with SYBR (Thermo Fisher) as well as specific primers and then subjected to qRT-PCR. The primers were provided by Sangon (Shanghai, China) and listed as follows: SNHG3: Forward, 5'-ACCTAGTCTCAGGTGGGTCC-3'; Reverse, 5'-CAACCTCCCGTTGCTACCAT-3'; YAP1: Forward, 5'-ACTGCTTCGGCAGGAGTTAG-3'; Reverse, 5'-GGTTCGAGGGACACTGTAGC-3'; miR-340-5p: Forward, 5'-GCGGTTATAAAGCAATGAGA-3'; Reverse, 5'-GTGCGTGTCGTGGAGTCG-3'; glyceraldehyde3-phosphate dehydrogenase (GAPDH): (Forward, 5' - CGGATTTGGTCGTATTGGGC-3'; Reverse,
5'-CCCGTTCTCAGCCATGTAGTT-3'); U6: (Forward, 5'-GCTTCGGCAGCACATATACTAAAAT-3'; Reverse, 5'-CGCTTCACGAATTTGCGTGTCAT-3'). GAPDH and U6 were regarded as endogenous controls. The relative RNA expression was analyzed by $2^{-\Delta \Delta C t}$ method [24].

Western blot. The total protein was extracted via the lysis buffer (Solarbio) with a $1 \%$ protease inhibitor. The lysates were centrifuged and then the supernatant was harvested for protein quantification using a bicinchoninic acid protein assay kit (Beyotime). After the denaturation at $100^{\circ} \mathrm{C}$ for $5 \mathrm{~min}, 20 \mu \mathrm{g}$ protein was loaded and separated by sodium dodecyl sulfate-polyacrylamide gel electrophoresis, followed by transfer of polyvinylidene difluoride membranes (Millipore, Billerica, MA, USA). The transferred membranes were incubated in the blocking buffer (Beyotime) for $30 \mathrm{~min}$ and then interacted with the indicated primary antibodies overnight and corresponding secondary antibody for $2 \mathrm{~h}$. The antibodies were provided by Abcam (Cambridge, UK) and listed as follows: anti-YAP1 (ab81183, 1:1000 dilution), anti-B-cell leukemia/lymphoma 2 (Bcl-2) (ab196495, 1:1000 dilution), anti- $\beta$-catenin (ab2365, 1:1000 dilution), anti-cMyc (ab39688, 1:500 dilution), anti-E-cadherin (ab15148, 1:500 dilution), anti-GAPDH (ab9485, 1:3000 dilution) and horseradish peroxidase-labeled IgG (ab6721, 1:10000 dilution). The protein blots were developed by Enhanced Chemiluminescence Western Blotting Substrate (Solarbio) and the relative protein level was analyzed by QuantityOne software (Bio-Rad, Hercules, CA, USA) and normalized to GAPDH level.

Cell transfection. The overexpression vector of YAP1 was constructed by cloning its full-length sequences into the pcDNA3.1 vector (Thermo Fisher) and the pcDNA3.1 empty vector was regarded as a negative control (pcDNA). The short hairpin RNA (shRNA) for SNHG3 (sh-SNHG3, 5'CUAGGAAUGCACAUUCUUUC-3'), shRNA for YAP1 (sh-YAP1，5'-AGGCCUCCUCUUCCTGAUGGAUU-3'), shRNA negative control (sh-NC, 5'-UUCUCCGAACGUGUCACGU-3'), miR-340-5p mimic (miR-340-5p, 5'-UUAUAAAGCAAUGAGACUGAUU-3'), mimic negative control (miR-NC, 5'-UUCUCCGAACGUGUCACGUUU-3'), miR-340-5p inhibitor (anti-miR-340-5p, 5'-AAUCAGUCUCAUUGCUUUAUAA-3'), inhibitor negative control (anti-miR-NC, 5'-CAGUACUUUUGUGUAGUACAA-3') were synthesized by GenoMeditech (Shanghai, China). TU177 and AMC-HN-8 cells were transfected with these vectors or oligonucleotides via the Lipofectamine $^{\mathrm{Tm}} 2000$ transfection reagent (Thermo Fisher) for $24 \mathrm{~h}$.

3-(4,5-dimethylthiazol-2-yl)-2,5-diphenyl-tetrazolium bromide (MTT). TU177 and AMC-HN- 8 cells $\left(4 \times 10^{3}\right.$ cells per well) were plated into 96-well plates. After incubation at $37^{\circ} \mathrm{C}$ for $0 \mathrm{~h}, 24 \mathrm{~h}, 48 \mathrm{~h}$, and $72 \mathrm{~h}, 10 \mu \mathrm{l}$ MTT solution (Beyotime) was added into each well, and cells continued the incubation for $4 \mathrm{~h}$. Next, $100 \mu \mathrm{l}$ dimethyl sulfoxide was applied to dissolve the formed formazan. The optical density 
(OD) value at $490 \mathrm{~nm}$ was examined through a microplate reader (Bio-Rad).

Flow cytometry. The cell apoptotic rate was measured with flow cytometry via the Apoptosis Detection Kit (Beyotime). TU177 and AMC-HN- 8 cells $\left(1 \times 10^{5}\right.$ cells per well) were added into 12 -well plates and maintained for $72 \mathrm{~h}$. Subsequently, cells were harvested, washed with phosphate buffer saline, resuspended in binding buffer, and then stained with Annexin V-fluorescein isothiocyanate (FITC) and propidium iodide (PI) (Solarbio) for $15 \mathrm{~min}$. The cells were examined with a flow cytometer (BD, San Jose, CA, USA) and the apoptotic rate was presented as the percentage of cells at upper and lower right quadrants.

Glucose utilization, oxygen consumption, lactate production, and pyruvate level. Glycolysis was assessed via glucose utilization, oxygen consumption, lactate production, and pyruvate level. TU177 and AMC-HN- 8 cells $\left(3 \times 10^{5}\right.$ cells per well) were plated into 6-well plates and incubated for $72 \mathrm{~h}$. The medium was collected for detection of glucose utilization, lactate production, and pyruvate level using a specific assay kit (Biovision, Milpitas, CA, USA) following the manufacturer's protocols. The oxygen consumption was measured by the XFe 96 Extracellular Flux Analyzer (Seahorse Bioscience, Billerica, MA, USA) as previously reported [25].

Dual-luciferase reporter assay. The complementary sequences of miR-340-5p and SNHG3 or YAP1 were searched by starBase (http://starbase.sysu.edu.cn/index. php). The SNHG3 sequences or 3' untranslated region (UTR) of YAP1 containing binding sites (CUUUAUA) of miR-340-5p were cloned into the downstream of the luciferase gene in the psiCHECK-2 vector (Promega, Madison, WI, USA) to form wild-type (WT) luciferase reporter vectors WT-SNHG3 or YAP1 3'UTR-WT, respectively. Their mutants (MUT) MUT-SNHG3 and YAP1 3'UTR-MUT were generated by mutating the seeded sites to GAAAUAU. For dual-luciferase reporter assay, these WT or MUT constructs and miR-NC or miR-340-5p were co-transfected into TU177 and AMC-HN-8 cells using Lipofectamine ${ }^{\mathrm{TM}} 2000$ transfection reagent for $48 \mathrm{~h}$. Subsequently, luciferase activity was examined by luciferase assay kit (GeneCopoeia, Rockville, MD, USA).

Xenograft model. The animal research was approved by the ethics committee of the Zhoukou Central Hospital and was performed under the experimental animal use guidelines of the National Institutes of Health. Five-weekold male $\mathrm{BALB} / \mathrm{c}$ nude mice were obtained from Charles River (Beijing, China) and subcutaneously injected with AMC-HN- 8 cells $\left(2 \times 10^{6}\right.$ cells/mouse $)$ stably transfected with sh-NC, sh-SNHG3 or sh-SNHG3 + anti-miR-340-5p ( $n=6$ per group). The tumor volume was measured four days after the cell injection for one week and calculated with a formula: volume $=$ length $\times$ width $^{2} / 2$. After 27 days, the mice were euthanized and tumors were collected. The tumor weight and expression of SNHG3, miR-340-5p, and YAP1 were measured.
Statistical analysis. The data were expressed as mean \pm standard deviation (S.D.) from 3 repeats. Statistical analysis was carried out by GraphPad Prism 7 software (GraphPad Inc., La Jolla, CA, USA). The linear association of the levels of YAP1-SNHG3, miR-340-5p-SNHG3, or miR-340$5 \mathrm{p}$-YAP1 was analyzed by Spearman's correlation coefficient. The difference was analyzed via Student's t-test or one-way analysis of variance (ANOVA) with Tukey's test, and was significant when $\mathrm{p}<0.05$.

\section{Results}

The expression levels of SNHG3 and YAP1 are increased in LSCC tissues and cells. To explore the potential role of SNHG3 and YAP1 in LSCC development, the expression levels of SNHG3 and YAP1 were measured in 25 LSCC tissues. As shown in Figure 1A, the expression of SNHG3 was significantly upregulated in LSCC tissues compared with that in corresponding normal samples. Moreover, the mRNA and protein levels of YAP1 were markedly higher in LSCC tissues than those in the normal group (Figures 1B, 1C). Meanwhile, there was a positive correlation between the levels of YAP1 mRNA and SNHG3 in LSCC tissues $(r=0.6893, p=0.0002$, Figure 1D). These results suggested that dysregulated SNHG3 and YAP1 might be involved in the progression of LSCC.

The knockdown of SNHG3 represses cell viability and glycolysis but promotes apoptosis in LSCC cells. To investigate the role of SNHG3 in LSCC progression, the abundance of this IncRNA was measured in LSCC cell lines and then knocked down using shRNA for SNHG3. As displayed in Figures $2 \mathrm{~A}$ and $2 \mathrm{~B}$, the expression of $\mathrm{SNHG} 3$ was remarkably increased in TU177 and AMC-HN-8 cells compared with that in NP69 cells, and its abundance was effectively reduced more than $54 \%$ in TU177 and AMC-HN-8 cells by introduction of sh-SNHG3. Furthermore, the knockdown of SNHG3 obviously decreased cell viability at $48 \mathrm{~h}$ and $72 \mathrm{~h}$ in the two cell lines (Figures 2C, 2D). In addition, the data of flow cytometry described that silence of SNHG3 induced 18\% and $20 \%$ of the apoptotic rate in TU177 and AMC-HN-8 cells, respectively (Figure 2E). Besides, the interference of SNHG3 significantly increased oxygen consumption but decreased glucose utilization and the levels of lactate and pyruvate in TU177 and AMC-HN-8 cells (Figures 2F-2I). These findings indicated that SNHG3 knockdown inhibited LSCC progression in vitro.

The knockdown of YAP1 inhibits cell viability and glycolysis but increases apoptosis in LSCC cells. To assess the biological role of YAP1 in LSCC progression, the expression of YAP1 was detected in LSCC cell lines. As shown in Figures 3A-3C, the mRNA and protein levels of YAP1 were significantly elevated in TU177 and AMC-HN-8 cells in comparison to those in NP69 cells. Moreover, the functional assays were performed in TU177 and AMC-HN-8 cells with YAP1 knockdown. The knockdown efficacy was confirmed in TU177 and AMC-HN-8 cells transfected with sh-YAP1 
A
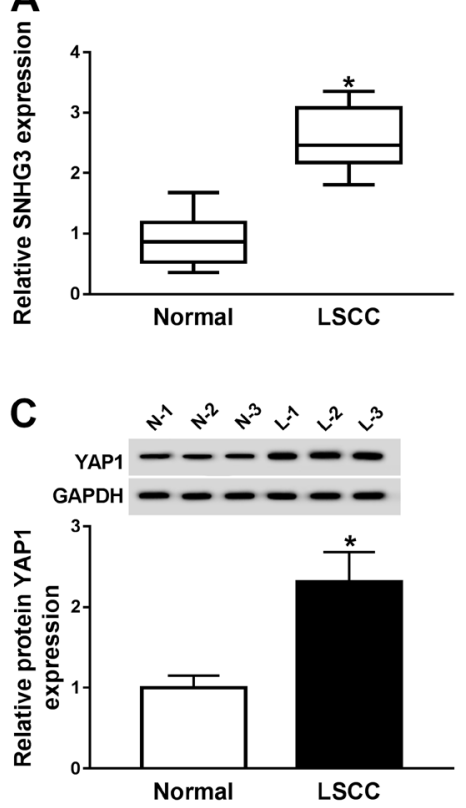

B

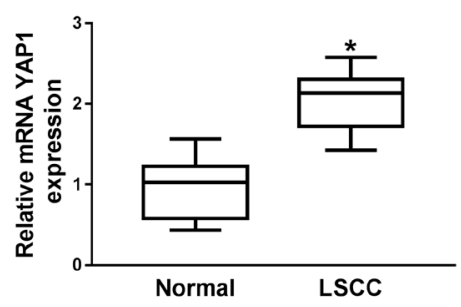

D

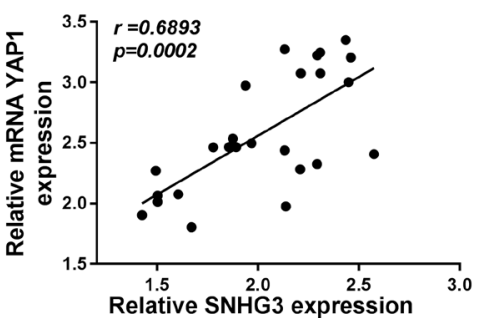

Figure 1. SNHG3 and YAP1 expression levels are enhanced in LSCC tissues. A, B) The expression levels of SNHG3 and YAP1 mRNA were measured in LSCC tissues $(n=25)$ and normal samples by qRT-PCR. C) The protein level of YAP1 was detected in LSCC tissues and normal samples by western blot. D) The correlation between the levels of YAP1 mRNA and SNHG3 in LSCC tissues was analyzed. ${ }^{\star} \mathbf{p}<0.05$
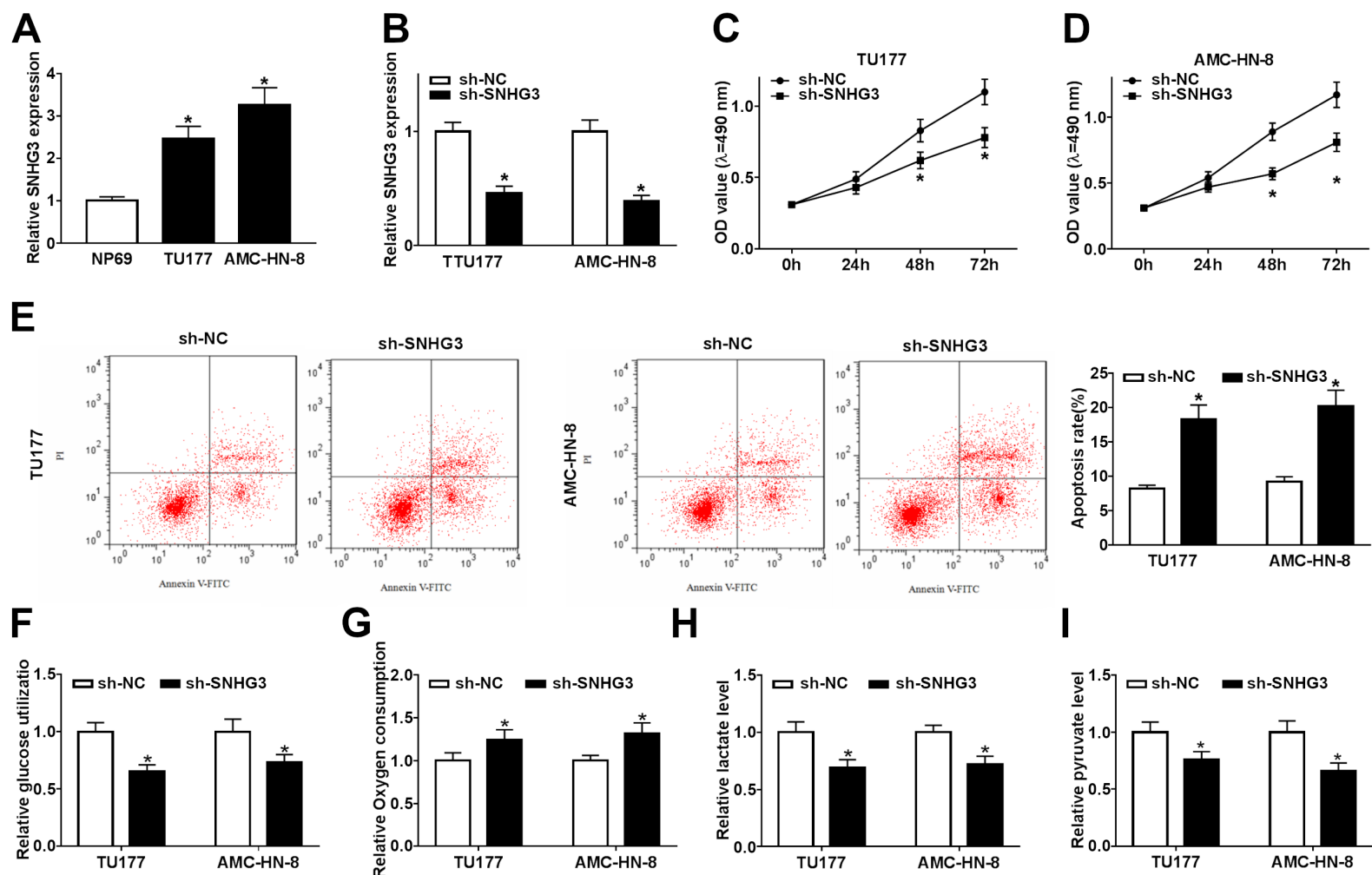

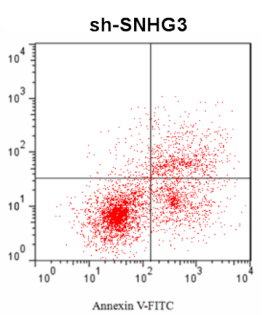

$\mathbf{G}$

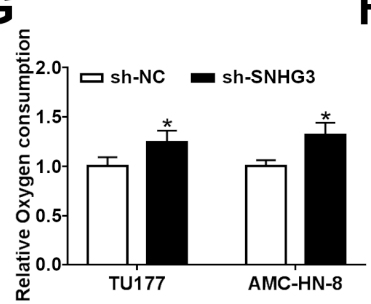

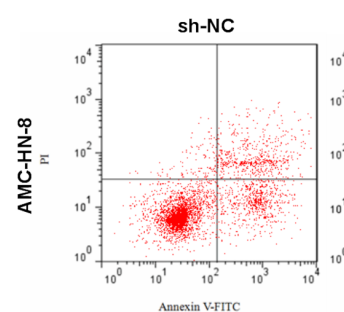

H

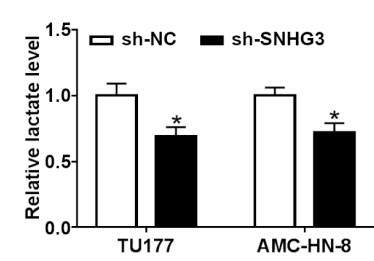

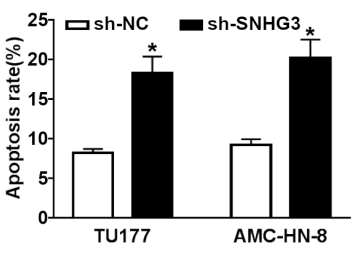

I

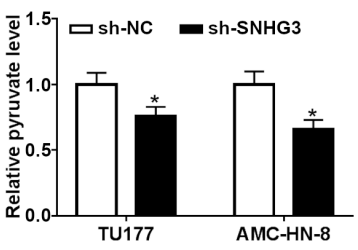

Figure 2. The knockdown of SNHG3 inhibits cell viability and glycolysis but induces apoptosis in LSCC cells. A) The expression of SNHG3 was measured in LSCC cell lines (TU177 and AMC-HN-8) and control (NP69) by qRT-PCR. B) The abundance of SNHG3 was detected in TU177 and AMCHN-8 cells transfected with sh-SNHG3 or sh-NC by qRT-PCR. C, D) Cell viability was measured in TU177 and AMC-HN-8 cells transfected with sh-SNHG3 or sh-NC at $0 \mathrm{~h}, 24 \mathrm{~h}, 48 \mathrm{~h}$, and $72 \mathrm{~h}$ by MTT. E) Cell apoptosis was detected in TU177 and AMC-HN-8 cells transfected with sh-SNHG3 or sh-NC at $72 \mathrm{~h}$ by flow cytometry. F-I) The relative glucose utilization, oxygen consumption, lactate production, and pyruvate level were examined in TU177 and AMC-HN-8 cells transfected with sh-SNHG3 or sh-NC at 72 h by specific kit. ${ }^{*} \mathrm{p}<0.05$ 
with more than $60 \%$ reduction in the abundance of YAP1 at mRNA and protein levels compared with the sh-NC group (Figures 3D, 3E). Additionally, the knockdown of YAP1 evidently declined the viability of TU177 and AMC-HN-8 cells at $48 \mathrm{~h}$ and $72 \mathrm{~h}$ (Figures 3F, 3G). Furthermore, the downregulation of YAP1 resulted in an approximately $20 \%$ apoptotic rate in TU177 and AMC-HN-8 cells (Figure $3 \mathrm{H}$ ). Besides, the silence of YAP1 induced the obviously decreased glucose utilization and the levels of lactate and pyruvate as well as increased oxygen consumption in the two LSCC cell lines (Figures 3I-3L, Supplementary Figures S1A-S1D). These data uncovered that YAP1 silence suppressed LSCC development in vitro.

YAP1 overexpression attenuates the effect of SNHG3 knockdown on cell viability, apoptosis, and glycolysis in LSCC cells. To explore whether YAP1 was involved in SNHG3-mediated LSCC progression in vitro, the effect of SNHG3 on YAP1 expression was investigated in LSCC cells. The results showed that the abundances of YAP1 at mRNA and protein levels were significantly reduced by knockdown of SNHG3 in TU177 and AMC-HN-8 cells (Figures 4A-4D). Moreover, the rescue experiments were performed in TU177 and AMC-HN-8 cells transfected with sh-NC, sh-SNHG3, sh-SNHG3, and pcDNA or YAP1. The data of MTT displayed that overexpression of YAP1 reversed knockdown of SNHG3mediated viability inhibition in TU177 and AMC-HN-8 cells (Figures 4E, 4F). In addition, knockdown of SNHG3-induced apoptosis of the two cell lines was weakened by the introduction of YAP1 (Figures 4G, 4H). Furthermore, the addition of YAP1 abrogated the effect of SNHG3 silence on glucose utilization, oxygen consumption, and the levels of lactate and pyruvate in TU177 and AMC-HN-8 cells (Figures 4I-4L). These results suggested that SNHG3 regulated YAP1 expression to mediate LSCC progression in vitro.

SNHG3 sponges miR-340-5p to regulate YAP1 expression in LSCC cells. To explore how SNHG3 regulates YAP1 expression, the potential miRNAs were explored. starBase online tool predicted that there were binding sites between SNHG3 and miR-340-5p (Figure 5A). To confirm the target association between SNHG3 and miR-340-5p, WT-SNHG3 and MUT-SNHG3 were constructed and dual-luciferase reporter assay was performed in TU177 and AMC-HN-8 cells. Overexpression of miR-340-5p led to approximately $50 \%$ loss of luciferase activity in the WT-SNHG3 group, while it showed little effect on the activity in the MUT-SNHG3 group (Figures 5B, 5C). Meanwhile, YAP1 also had the binding sites of miR-340-5p, as predicted by starBase (Figure 5D). To validate the association between miR-340-5p
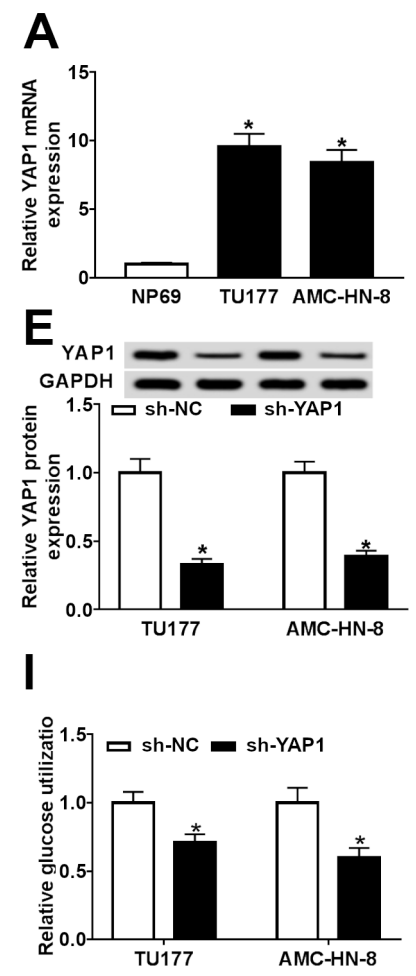

B

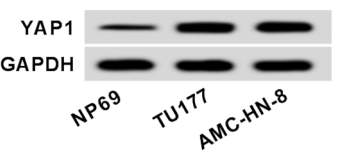

$\mathbf{F}$
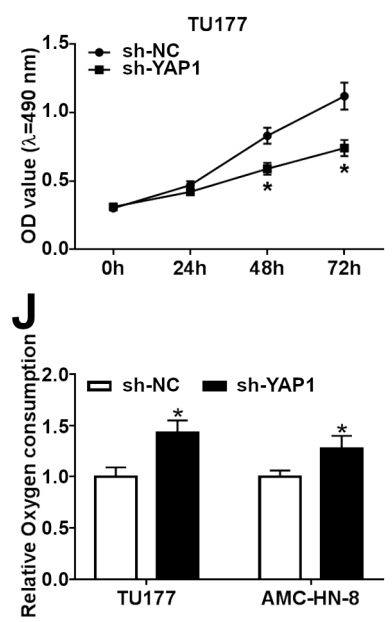

C

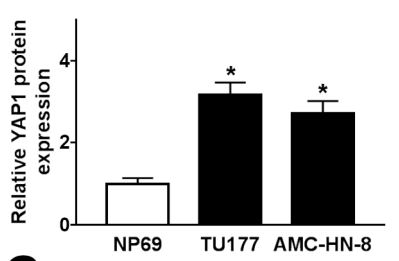

G
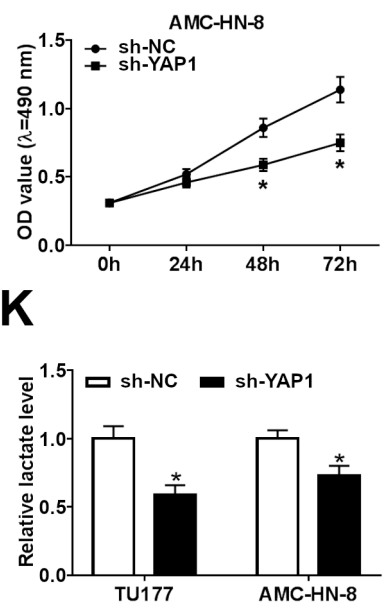

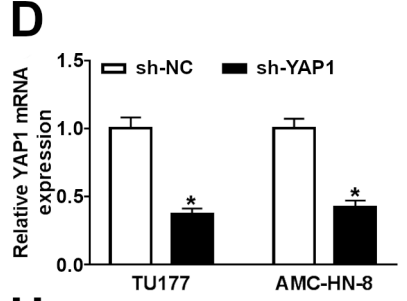

$\mathrm{H}$

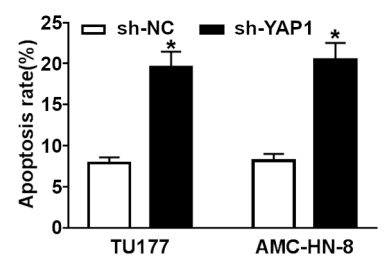

$\mathbf{L}$

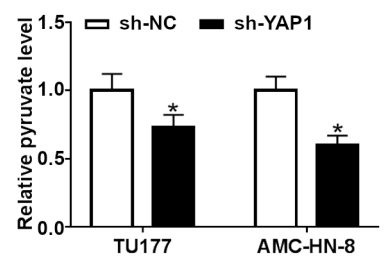

Figure 3. The interference of YAP1 suppresses cell viability and glycolysis but promotes apoptosis in LSCC cells. A-C) The mRNA and protein levels of YAP1 were measured in LSCC cell lines (TU177 and AMC-HN-8) and control (NP69) by qRT-PCR and western blot. D, E) The abundances of YAP1 mRNA and protein were detected in TU177 and AMC-HN-8 cells transfected with sh-YAP1 or sh-NC by qRT-PCR and western blot. F, G) Cell viability was measured in TU177 and AMC-HN-8 cells transfected with sh-YAP1 or sh-NC at $0 \mathrm{~h}, 24 \mathrm{~h}, 48 \mathrm{~h}$, and $72 \mathrm{~h}$ by MTT. H) Cell apoptosis was detected in TU177 and AMC-HN-8 cells transfected with sh-YAP1 or sh-NC at $72 \mathrm{~h}$ by flow cytometry. I-L) The relative glucose utilization, oxygen consumption, lactate production, and pyruvate level were examined in TU177 and AMC-HN-8 cells transfected with sh-YAP1 or sh-NC at $72 \mathrm{~h}$ by specific kit. ${ }^{*} \mathrm{p}<0.05$ 


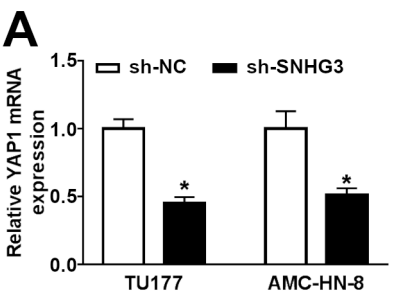

E

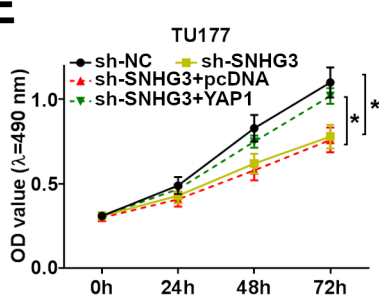

I

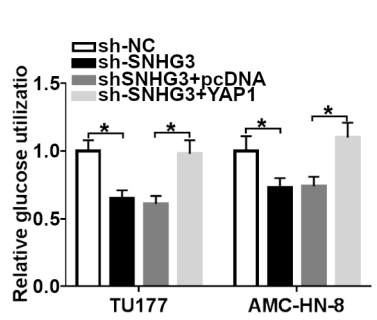

B

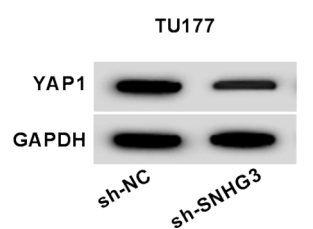

$\mathbf{F}$
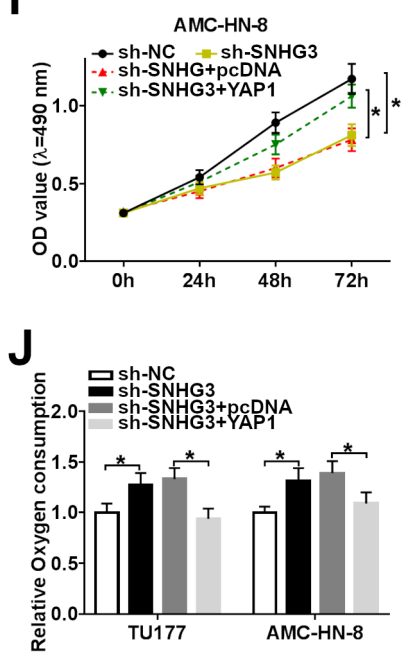

C

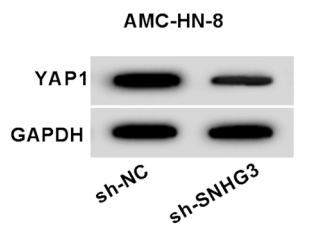

G
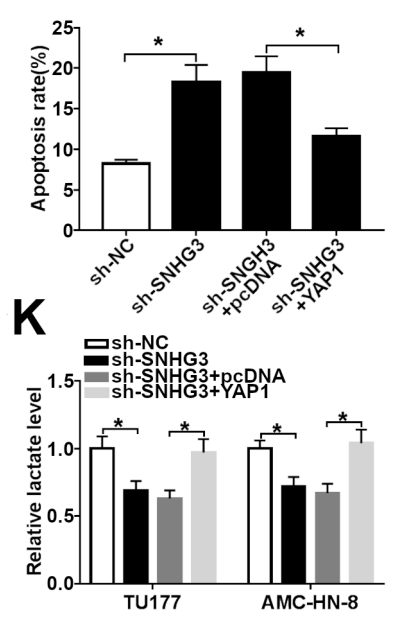

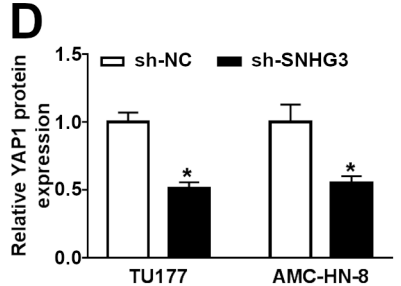

H
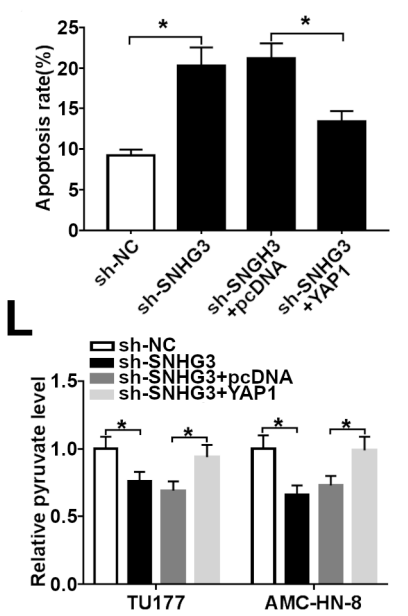

Figure 4. YAP1 overexpression reverses the effect of SNHG3 knockdown on cell viability, apoptosis, and glycolysis in LSCC cells. A-D) The mRNA and protein levels of YAP1 were measured in TU177 and AMC-HN-8 cells transfected with sh-SNHG3 or sh-NC by qRT-PCR and western blot. E, F) Cell viability was detected in TU177 and AMC-HN-8 cells transfected with sh-NC, sh-SNHG3, sh-SNHG3, and pcDNA or YAP1 at 0 h, 24 h, 48 h, and 72 h by MTT. G, H) Cell apoptosis was measured in TU177 and AMC-HN-8 cells transfected with sh-NC, sh-SNHG3, sh-SNHG3, and pcDNA or YAP1 at $72 \mathrm{~h}$ by flow cytometry. I-L) The relative glucose utilization, oxygen consumption, lactate production, and pyruvate level were examined in TU177 and AMC-HN-8 cells transfected with sh-NC, sh-SNHG3, sh-SNHG3, and pcDNA or YAP1 at 72 h by specific kit. ${ }^{*}$ p $<0.05$

and YAP1, YAP1 3'UTR-WT and YAP1 3'UTR-MUT were generated and transfected in TU177 and AMC-HN-8 cells. The results of dual-luciferase reporter assay revealed that the luciferase activity in the YAP1 3'UTR-WT group was notably decreased by miR-340-5p overexpression, whereas it was not changed in the YAP1 3'UTR-MUT group (Figures 5E, 5F). Furthermore, the effects of SNHG3 and miR-340-5p on YAP1 expression were analyzed. The data of western blot displayed that the protein level of YAP1 was significantly reduced by SNHG3 knockdown and restored by miR-340-5p inhibition in TU177 and AMC-HN-8 cells (Figures 5G, 5H). Moreover, miR-340-5p expression was evidently enhanced by SNHG3 knockdown (Supplementary Figure S2). These data indicated that SNHG3 could regulate YAP1 expression by sponging miR-340-5p in LSCC cells.

Overexpression of miR-340-5p suppresses cell viability and glycolysis but induces apoptosis in LSCC cells. To explore the role of miR-340-5p in LSCC progression, its expression was measured in LSCC tissues and cells. As displayed in Figures 6A and 6B, the level of miR-340-5p was significantly decreased in LSCC tissues and cell lines compared with their corresponding controls. Meanwhile, the expression of miR-340-5p in LSCC tissues was negatively correlated with SNHG3 ( $\mathrm{r}=-0.6052, \mathrm{p}=0.0013)$ and YAP1 $(r=-0.6889, p=0.0001$, Figures $6 C, 6 D)$. To investigate the biological role of miR-340-5p, its abundance was overexpressed by miR-340-5p mimic and knocked down by antimiR-340-5p in TU177 and AMC-HN-8 cells (Figure 6E). Moreover, overexpression of miR-340-5p obviously decreased the viability of TU177 and AMC-HN-8 cells at $48 \mathrm{~h}$ and $72 \mathrm{~h}$ (Figures 6F, 6G). In addition, the upregulation of miR-340-5p induced significant apoptosis in the two cell lines (Figure 6H). Besides, abundant expression of miR-340-5p significantly repressed glucose utilization and the levels of lactate and pyruvate but promoted oxygen consumption in TU177 and AMC-HN-8 cells (Figures 6I-6L, Supplementary Figures S3A-S3D). Taken together, miR-340-5p suppressed LSCC progression in vitro.

The knockdown of SNHG3 restrains the Wnt/ $\beta$-catenin signaling by regulating miR-340-5p and YAP1 in LSCC cells. To explore the potential signaling pathway implicated in this study, the expression of a protein associated with the Wnt/ $\beta$-catenin pathway was measured in TU177 and AMC-HN-8 cells transfected with sh-NC, sh-SNHG3, sh-SNHG3 and anti-miR-NC, anti-miR-340-5p, pcDNA or YAP1. The results of western blot showed that the protein levels 

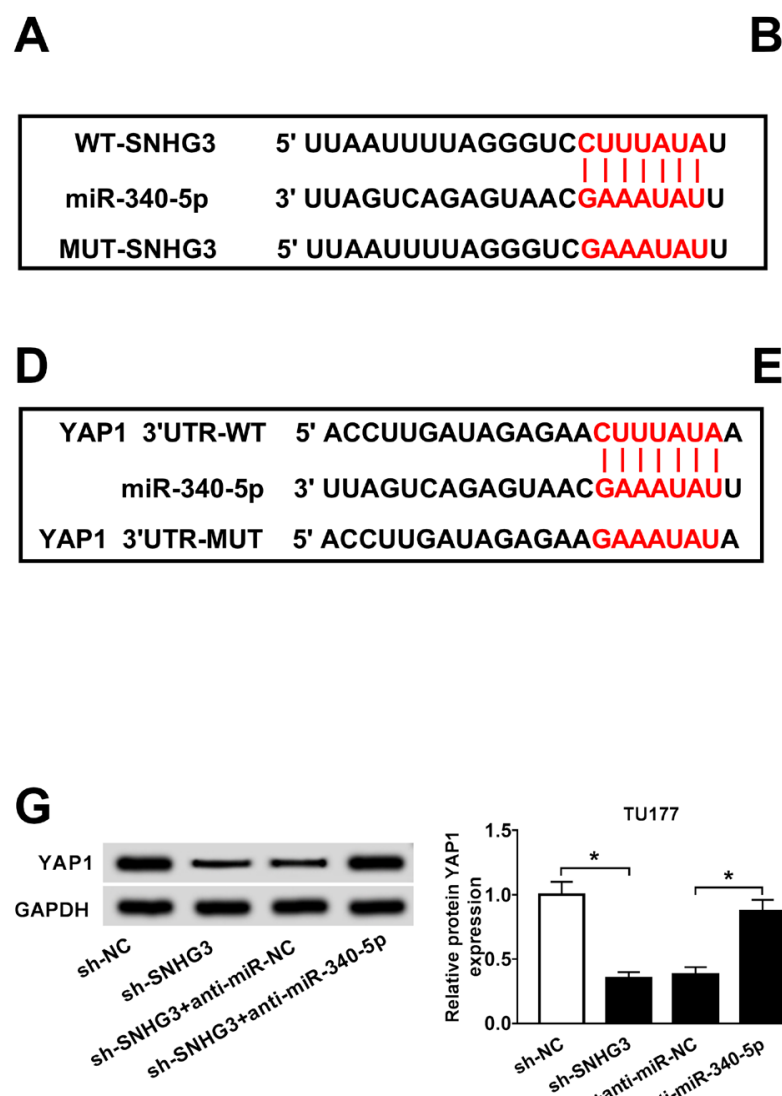

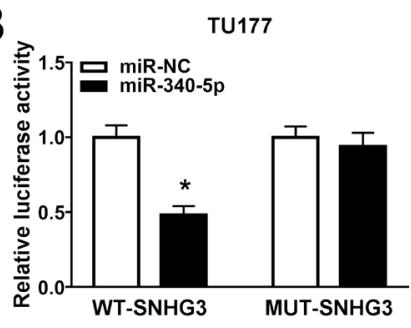

E

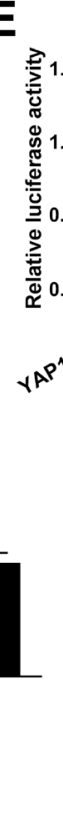

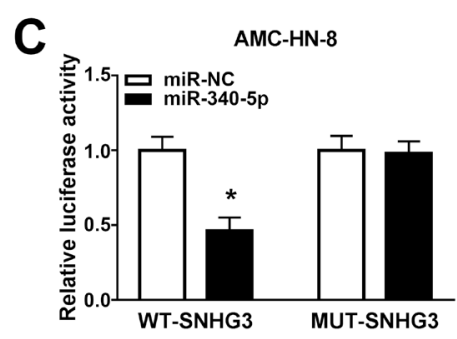

$\mathbf{F}$
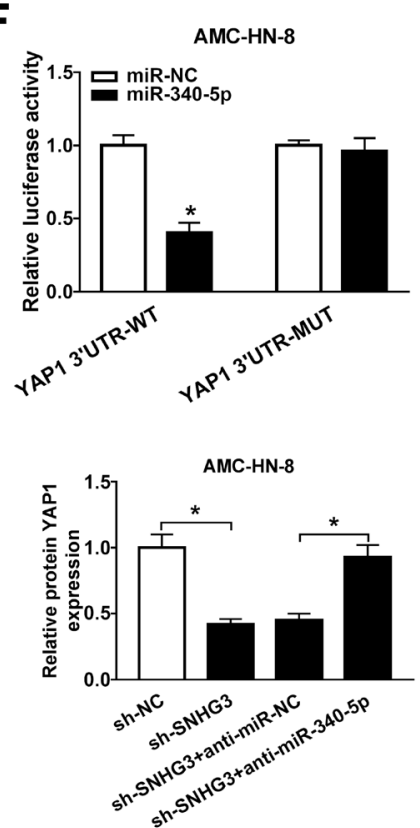

Figure 5. SNHG3 targets miR-340-5p to regulate YAP1 in LSCC cells. A) The binding sites of SNHG3 and miR-340-5p were predicted by starBase. B, C) Luciferase activity was measured in TU177 and AMC-HN-8 cells co-transfected with WT-SNHG3 or MUT-SNHG3 and miR-NC or miR-340-5p. D) The binding sites of miR-340-5p and YAP1 were predicted by starBase. E, F) Dual-luciferase reporter assay was performed in TU177 and AMC-HN-8 cells co-transfected with YAP1 3'UTR-WT or YAP1 3'UTR-MUT and miR-NC or miR-340-5p. G, H) The expression of YAP1 protein was measured in TU177 and AMC-HN-8 cells transfected with sh-NC, sh-SNHG3, sh-SNHG3, and anti-miR-NC or anti-miR-340-5p by western blot. ${ }^{*} \mathrm{p}<0.05$

of $\beta$-catenin, c-Myc, and Bcl-2 were significantly declined and E-cadherin protein expression was notably enhanced by the knockdown of SNHG3 in TU177 and AMC-HN-8 cells, while these events were abated by miR-340-5p knockdown or YAP1 overexpression (Figures $7 \mathrm{~A}, 7 \mathrm{~B}$ ). These findings indicated that silence of SNHG3 inhibited the Wnt/ $\beta$-catenin pathway by regulating miR-340-5p and YAP1 in LSCC cells.

The knockdown of SNHG3 reduces LSCC xenograft tumor growth by regulating $\mathrm{miR}-340-5 \mathrm{p}$. To further explore the role of SNHG3 in LSCC progression in vivo, AMC-HN-8 cells stably transfected with sh-NC, sh-SNHG3, or sh-SNHG3 + anti-miR-340-5p were used to establish murine xenograft model, named as sh-NC, sh-SNHG3 or sh-SNHG3 + antimiR-340-5p group, respectively. As displayed in Figure 8A and $8 \mathrm{~B}$, tumor volume and weight were significantly decreased in the sh-SNHG3 group compared with those in the sh-NC group. Moreover, the expression levels of SNHG3, miR-340-5p, and YAP1 were measured in the tumor tissues. The results showed that the expression levels of SNHG3 and YAP1 mRNA and protein were obviously decreased but the miR-340-5p expression was notably increased in the tumor tissues of the sh-SNHG3 group when compared to those in the sh-NC group (Figures 8C, 8D). However, these events were weakened via miR-340-5p downregulation in the sh-SNHG3 + anti-miR-340-5p group compared with those in the sh-SNHG3 group (Figures 8A-8D). These results indicated the anti-cancer role of SNHG3 knockdown in LSCC in vivo. A graphic summary of the SNHG3/ miR-340-5p/YAP1/Wnt/ $\beta$-catenin pathway in LSCC development is exhibited in Figure 9.

\section{Discussion}

The competitive endogenous RNA (ceRNA) networks of lncRNA/miRNA/mRNA have vital roles in the development and treatment of LSCC [26]. Based on previous demonstrations, there are many dysregulated lncRNAs involved in the development of LSCC, such as nuclear paraspeckle assembly 

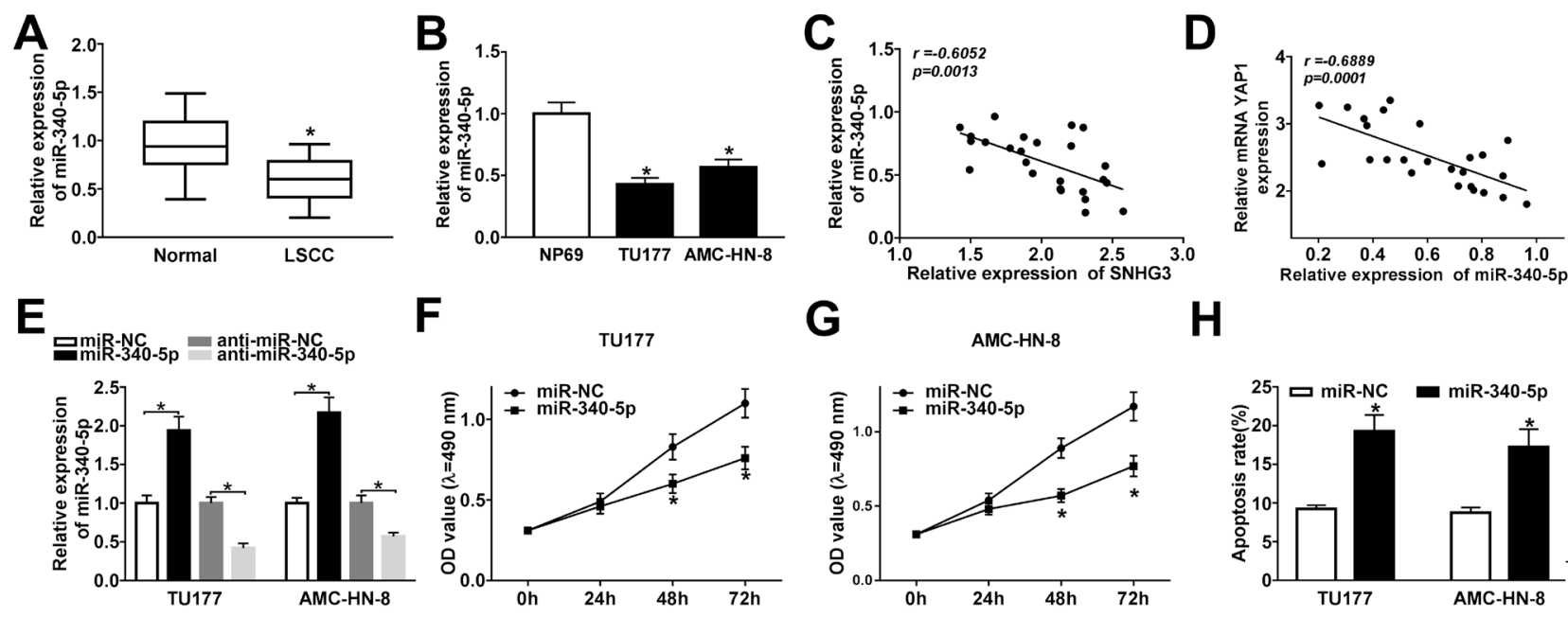

G
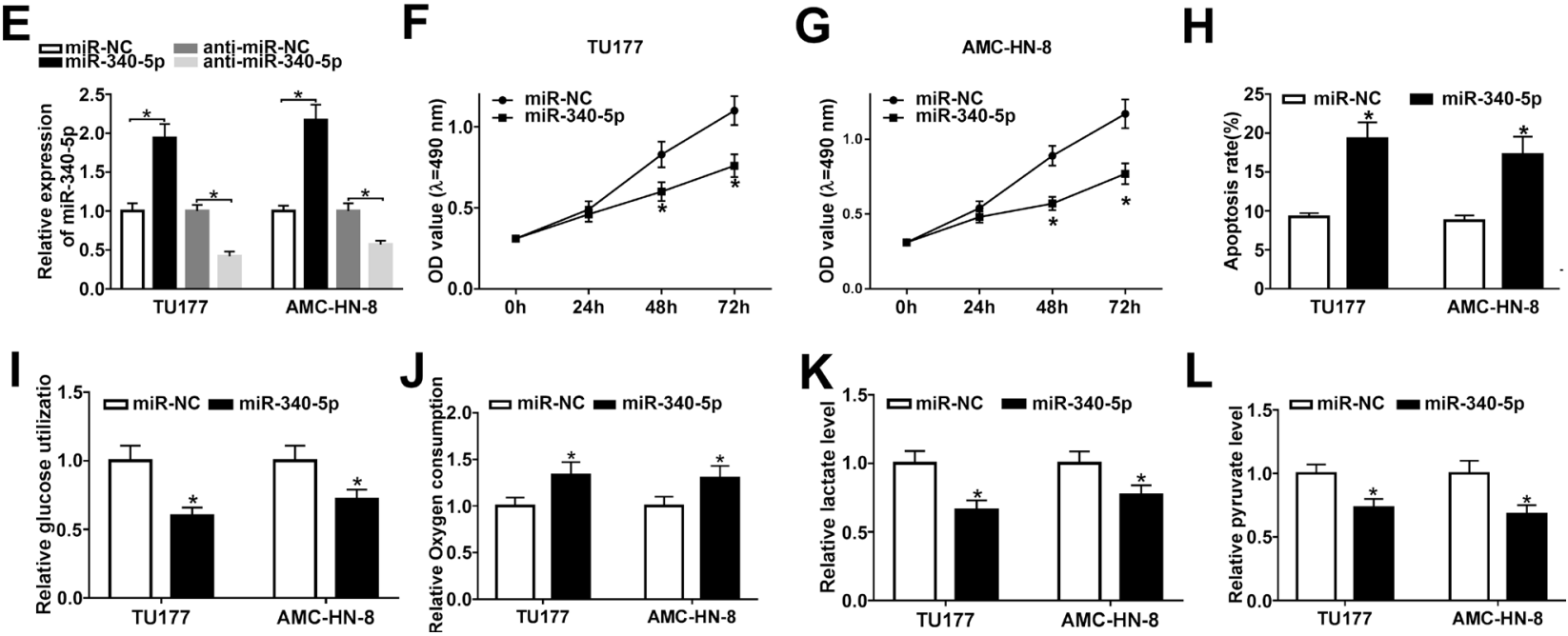

Figure 6. miR-340-5p overexpression inhibits cell viability and glycolysis but induces apoptosis in LSCC cells. A, B) The expression of miR-340-5p was measured in LSCC tissues and cell lines by qRT-PCR. C, D) The correlation between the levels of miR-340-5p and SNHG3 or YAP1 in LSCC tissues was assessed. E) The abundance of miR-340-5p was measured in TU177 and AMC-HN-8 cells transfected with miR-NC, miR-340-5p, anti-miR-NC, or anti-miR-340-5p by qRT-PCR. F, G) Cell viability was measured in TU177 and AMC-HN-8 cells transfected with miR-340-5p or miR-NC at 0 h, 24 h, $48 \mathrm{~h}$, and $72 \mathrm{~h}$ by MTT. H) Cell apoptosis was detected in TU177 and AMC-HN-8 cells transfected with miR-340-5p or miR-NC at 72 h by flow cytometry. I-L) The relative glucose utilization, oxygen consumption, lactate production, and pyruvate level were examined in TU177 and AMC-HN-8 cells transfected with miR-340-5p or miR-NC at $72 \mathrm{~h}$ by specific kit. ${ }^{*} \mathrm{p}<0.05$

transcript 1 (NEAT1), repulsive guidance molecule B antisense RNA 1 (RGMB-AS1) and zinc finger E-box-binding homeobox 2 antisense RNA 1 (ZEB2-AS1) [27-29]. Wang et al. showed that lncRNA SNHG3 could promote laryngeal carcinoma progression [11]. This report suggested that SNHG3 might serve as a promising target for the progression and treatment of LSCC. In the current research, we found that SNHG3 abundance was enhanced in LSCC, indicating that high expression of SNHG3 might be associated with the development of LSCC. To explore the function of SNHG3, we further performed the loss-of-function experiments and confirmed that SNHG3 knockdown inhibited LSCC cell viability and glycolysis but promoted apoptosis, which was also in agreement with that in other cell lines [10,30]. These data revealed the potential therapeutic role of SNHG3 inhibitor in LSCC.

The former studies indicated that SNHG3 could serve as a ceRNA for miR-326, miR-384, and miR-151a-3p in numerous types of cancers [7-9]. To figure out the ceRNA network of SNHG3 in our study, we were the first to confirm the target association of SNHG3-miR-340-5p and miR-340-5p-YAP1 in LSCC cells. Moreover, we found that
YAP1 expression was decreased by SNHG3 knockdown and restored by miR-340-5p inhibition, indicating that SNHG3 could derepress YAP1 expression by competitively binding with miR-340-5p. In this work, we found that miR-340-5p expression was declined in LSCC and could inhibit cell viability and induce apoptosis in LSCC cells, uncovering the anti-cancer role of miR-340-5p in LSCC, which was consistent with the former work [17]. Furthermore, our results displayed the inhibitive role of miR-340-5p in glycolysis in LSCC cells, which was similar to that in other types of cancer cells $[15,16,31]$. Meanwhile, miR-340-5p could be negatively regulated by SNHG3, indicating that miR-340-5p might act as a biomarker to monitor the influence of SNHG3 antagonism in LSCC. According to the published reports on YAP1 in LSCC $[20,32]$, we also found that high expression of YAP1 in LSCC contributed to LSCC malignancy by regulating cell viability, apoptosis, and glycolysis. Besides, we found that overexpression of YAP1 weakened the effect of SNHG3 knockdown on LSCC progression; indicating YAP1 was involved in the SNHG3-mediated mechanism. Collectively, SNHG3 acted as a ceRNA for miR-340-5p to regulate YAP1, mediating LSCC progression in vitro. Besides, we 

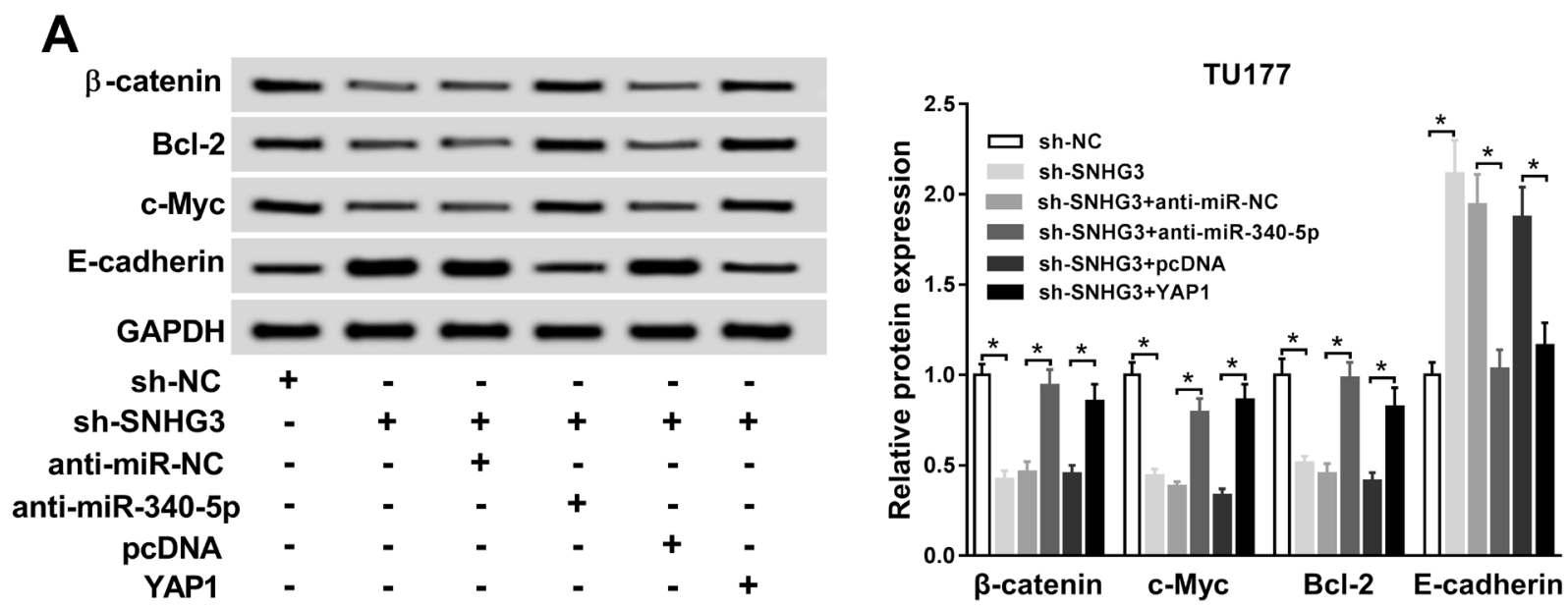

B
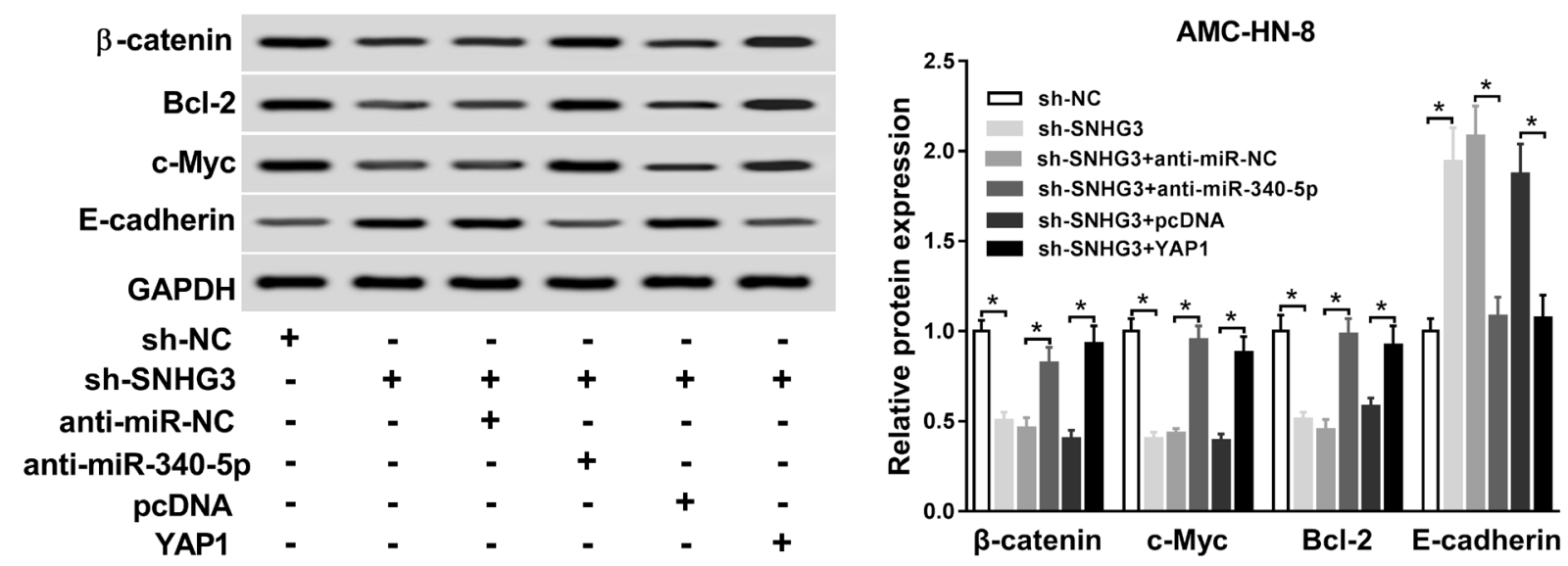

Figure 7. The knockdown of SNHG3 inhibits the Wnt/ $\beta$-catenin pathway by regulating miR-340-5p and YAP1 in LSCC cells. A, B) The protein levels of $\beta$-catenin, Bcl-2, c-Myc, and E-cadherin were determined in TU177 and AMC-HN-8 cells transfected with sh-NC, sh-SNHG3, sh-SNHG3, and antimiR-NC, anti-miR-340-5p, pcDNA or YAP1 by western blot. ${ }^{*} \mathrm{p}<0.05$

also validated the anti-cancer role of SNHG3 knockdown in LSCC in vivo by regulating miR-340-5p and YAP1 using a murine xenograft model.

Previous studies showed that $\mathrm{Wnt} / \beta$-catenin signaling was mediated by YAP1 $[21,33]$. Moreover, activation of the Wnt/ $\beta$-catenin signaling contributed to cell proliferation, migration, invasion, and glycolysis in LSCC $[23,34]$. Therefore, we next explored whether Wnt/ $\beta$-catenin signaling was associated with the SNHG3-mediated LSCC progression. $\mathrm{E}$-cadherin and $\beta$-catenin are the two key regulators of Wnt signaling [35]. The reduction of E-cadherin may activate Wnt signaling, promoting $\beta$-catenin entering the nucleus to induce the expression of downstream genes such as c-Myc and $\mathrm{Bcl}-2$, and then regulate cancer progression $[36,37]$. By detecting the abundances of $\beta$-catenin, E-cadherin, c-Myc, and Bcl-2 in LSCC cells, we found that knockdown of SNHG3 repressed the activation of the Wnt $/ \beta$-catenin signaling.
Moreover, knockdown of miR-340-5p and overexpression of YAP1 reversed the suppressive effect of SNHG3 silence on this pathway, indicating the inhibitive role of miR-340-5p and promoting role of YAP1 in the $\mathrm{Wnt} / \beta$-catenin signaling, which was also in agreement with the previous studies on the regulation of miR-340-5p and YAP1 on Wnt/ $\beta$-catenin pathway in other cancers $[38,39]$. Hence, we assumed that $\mathrm{SNHG} 3 / \mathrm{miR}-340-5 \mathrm{p} / \mathrm{YAP} 1$ regulated $\mathrm{Wnt} / \beta$-catenin signaling to mediate LSCC progression. However, the direct evidence in support of the interaction between $\mathrm{Wnt} / \beta$ catenin signaling and SNHG3-mediated LSCC progression should be provided in further study.

In conclusion, SHNG3 knockdown inhibited LSCC progression in vitro and in vivo, possibly by regulating miR-340-5p and YAP1. This study elucidated a new mechanism for understanding the pathogenesis of LSCC and indicated SNHG3 as a target for the treatment of LSCC. 

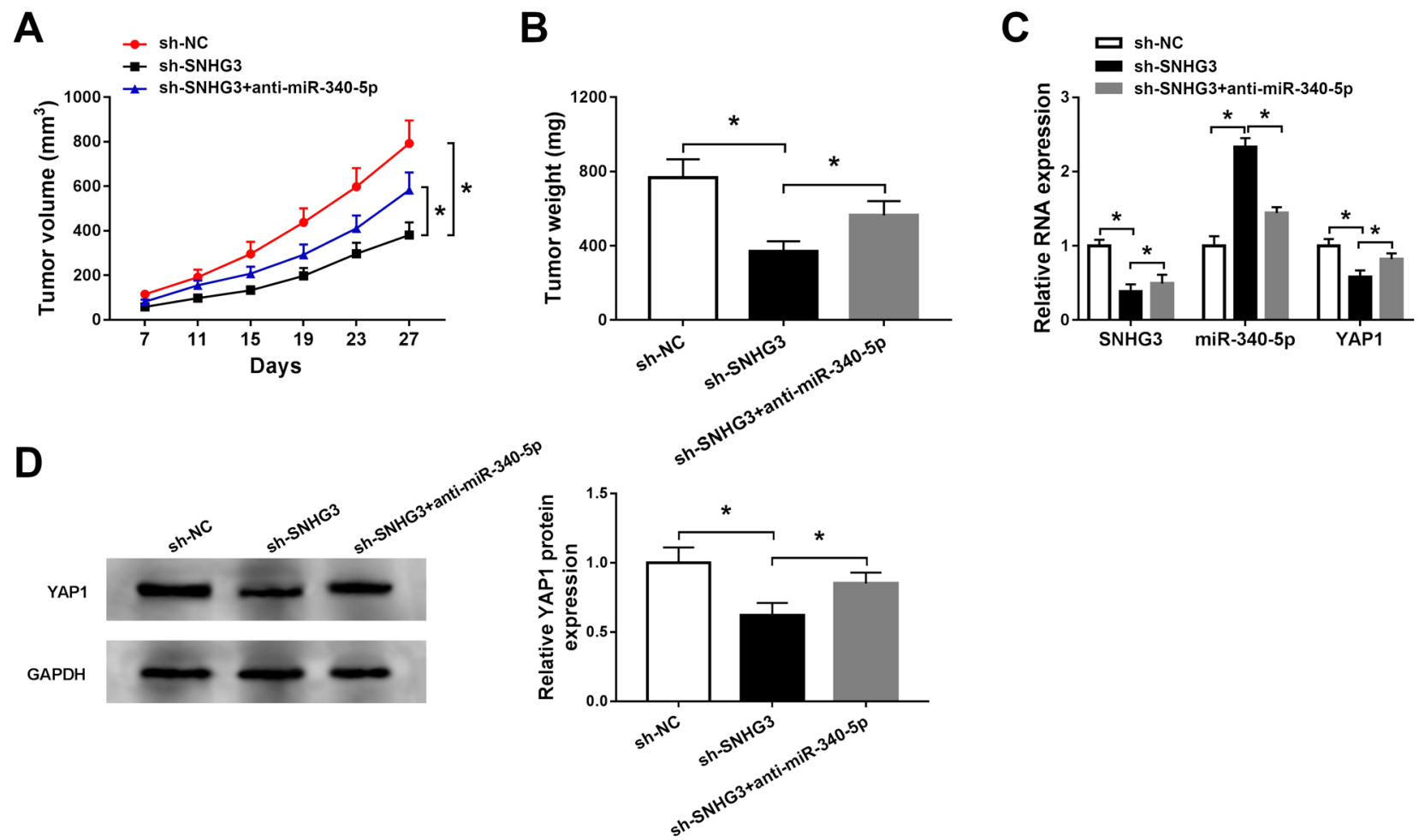

Figure 8. The knockdown of SNHG3 decreases xenograft tumor growth. A) Tumor volume was measured every 4 days from one week after cell injection. B) Tumor weight was measured in sh-NC, sh-SNHG3, and sh-SNHG3 + anti-miR-340-5p groups. C) The expression levels of SNHG3, miR-340-5p, and YAP1 were detected in the tumor tissues in sh-NC, sh-SNHG3, and sh-SNHG3 + anti-miR-340-5p groups by qRT-PCR. D) The expression of YAP1 protein was measured in the tumor tissues in sh-NC, sh-SNHG3, and sh-SNHG3 + anti-miR-340-5p groups by western blot. ${ }^{\star} p<0.05$

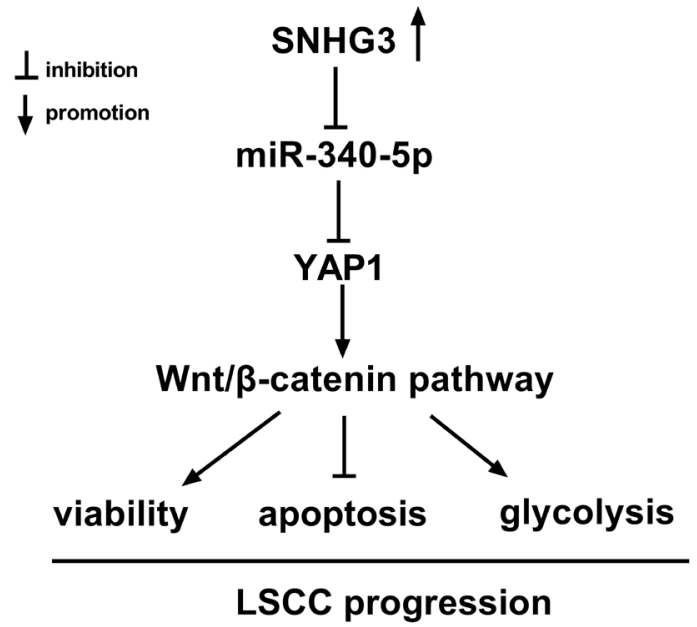

Figure 9. The graphic summary of the signaling axis in this study. SNHG3 expression was increased in LSCC. SNHG3 could regulate the miR-340$5 \mathrm{p} / \mathrm{YAP} 1$ axis to activate the $\mathrm{Wnt} / \boldsymbol{\beta}$-catenin pathway, which promoted cell viability and glycolysis and inhibited apoptosis.

Supplementary information is available in the online version of the paper.

\section{References}

[1] TOMEH C, HOLSINGER FC. Laryngeal cancer. Curr Opin Otolaryngol Head Neck Surg 2014; 22: 147-153. https://doi. org/10.1097/MOO.0000000000000032

[2] ALMADORI G, BUSSU F, CADONI G, GALLI J, PALUDETTI $G$ et al. Molecular markers in laryngeal squamous cell carcinoma: towards an integrated clinicobiological approach. Eur J Cancer 2005; 41: 683-693. https://doi. org/10.1016/j.ejca.2004.10.031

[3] OBID R, REDLICH M, TOMEH C. The Treatment of Laryngeal Cancer. Oral Maxillofac Surg Clin North Am 2019; 31: 1-11. https://doi.org/10.1016/j.coms.2018.09.001

[4] LUNT SY, VANDER HEIDEN MG. Aerobic glycolysis: meeting the metabolic requirements of cell proliferation. Annu Rev Cell Dev Biol 2011; 27: 441-464. https://doi. org/10.1146/annurev-cellbio-092910-154237

[5] BAYOUMI AS, SAYED A, BROSKOVA Z, TEOH JP, WILSON J et al. Crosstalk between Long Noncoding RNAs and MicroRNAs in Health and Disease. Int J Mol Sci 2016; 17: 356. https://doi.org/10.3390/ijms17030356

[6] COSSU AM, MOSCA L, ZAPPAVIGNA S, MISSO G, BOCCHETTI $\mathrm{M}$ et al. Long Non-coding RNAs as Important Biomarkers in Laryngeal Cancer and Other Head and Neck Tumours. Int J Mol Sci 2019; 20: 3444. https://doi.org/10.3390/ ijms20143444 
[7] ZHAO Q, WU C, WANG J, LI X, FAN Y et al. LncRNA SNHG3 Promotes Hepatocellular Tumorigenesis by Targeting miR-326. Tohoku J Exp Med 2019; 249: 43-56. https:// doi.org/10.1620/tjem.249.43

[8] MA Q, QI X, LIN X, LI L, CHEN L et al. LncRNA SNHG3 promotes cell proliferation and invasion through the miR384/hepatoma-derived growth factor axis in breast cancer. Hum Cell 2020; 33: 232-242. https://doi.org/10.1007/ s13577-019-00287-9

[9] ZHENG S, JIANG F, GE D, TANG J, CHEN H et al. LncRNA SNHG3/miRNA-151a-3p/RAB22A axis regulates invasion and migration of osteosarcoma. Biomed Pharmacother 2019; 112: 108695. https://doi.org/10.1016/j.biopha.2019.108695

[10] LI N, ZHAN X, ZHAN X. The lncRNA SNHG3 regulates energy metabolism of ovarian cancer by an analysis of mitochondrial proteomes. Gynecol Oncol 2018; 150: 343-354. https://doi.org/10.1016/j.ygyno.2018.06.013

[11] WANG L, SU K, WU H, LI J, SONG D. LncRNA SNHG3 regulates laryngeal carcinoma proliferation and migration by modulating the miR-384/WEE1 axis. Life Sci 2019; 232: 116597. https://doi.org/10.1016/j.lfs.2019.116597

[12] YANG G, YANG C, SHE Y, SHEN Z, GAO P. LINC01354 enhances the proliferation and invasion of lung cancer cells by regulating miR-340-5p/ATF1 signaling pathway. Artif Cells Nanomed Biotechnol 2019; 47: 3737-3744. https://doi. org/10.1080/21691401.2019.1667816

[13] KIM S, CHOI JY, SEOK HJ, PARK MJ, CHUNG HY et al. miR-340-5p Suppresses Aggressiveness in Glioblastoma Multiforme by Targeting Bcl-w and Sox2. Mol Ther Nucleic Acids 2019; 17: 245-255. https://doi.org/10.1016/j. omtn.2019.05.022

[14] RONGXIN S, PENGFEI L, LI S, XIAOCHEN J, YIHE H. MicroRNA-340-5p suppresses osteosarcoma development by down-regulating the Wnt/beta-catenin signaling pathway via targeting the STAT3 gene. Eur Rev Med Pharmacol Sci 2019; 23: 982-991. https://doi.org/10.26355/eurrev_201902_16985

[15] SUN Y, ZHAO X, ZHOU Y, HU Y. miR-124, miR-137 and miR-340 regulate colorectal cancer growth via inhibition of the Warburg effect. Oncol Rep 2012; 28: 1346-1352. https:// doi.org/10.3892/or.2012.1958

[16] YU C, WANG Y, PENG J, SHEN Q, CHEN M et al. Mitochondrial calcium uniporter as a target of microRNA-340 and promoter of metastasis via enhancing the Warburg effect. Oncotarget 2017; 8: 83831-83844. https://doi.org/10.18632/ oncotarget.19747

[17] YU W, ZHANG G, LU B, LI J, WU Z et al. MiR-340 impedes the progression of laryngeal squamous cell carcinoma by targeting EZH2. Gene 2016; 577: 193-201. https://doi. org/10.1016/j.gene.2015.11.045

[18] KOO JH, GUAN KL. Interplay between YAP/TAZ and Metabolism. Cell Metab 2018; 28: 196-206. https://doi. org/10.1016/j.cmet.2018.07.010

[19] PAN C, DU Z, CAI Z, LIU Y, SUN Y et al. Elevated expression of yesassociated protein is associated with the malignant status and prognosis of laryngeal squamous cell carcinoma. Mol Med Rep 2017; 16: 4934-4940. https://doi.org/10.3892/ mmr.2017.7187
[20] WANG L, SUN J, GAO P, SU K, WU H et al. Wnt1-inducible signaling protein 1 regulates laryngeal squamous cell carcinoma glycolysis and chemoresistance via the YAP1/TEAD1/ GLUT1 pathway. J Cell Physiol 2019; 234: 15941-15950. https://doi.org/10.1002/jcp.28253

[21] TOTARO A, PANCIERA T, PICCOLO S. YAP/TAZ upstream signals and downstream responses. Nat Cell Biol 2018; 20: 888-899. https://doi.org/10.1038/s41556-018-0142-z

[22] EL-SAHLI S, XIE Y, WANG L, LIU S. Wnt Signaling in Cancer Metabolism and Immunity. Cancers (Basel) 2019; 11: 904. https://doi.org/10.3390/cancers11070904

[23] LIU L, ZHANG P, SHAO Y, QUAN F, LI H. Knockdown of FOXJ1 inhibits the proliferation, migration, invasion, and glycolysis in laryngeal squamous cell carcinoma cells. J Cell Biochem 2019; 120: 15874-15882. https://doi.org/10.1002/ jcb. 28858

[24] LIVAK KJ, SCHMITTGEN TD. Analysis of relative gene expression data using real-time quantitative PCR and the 2(-Delta Delta C(T)) Method. Methods 2001; 25: 402-408. https://doi.org/10.1006/meth.2001.1262

[25] YANG X, ZHAO H, YANG J, MA Y, LIU Z et al. MiR-150$5 p$ regulates melanoma proliferation, invasion and metastasis via SIX1-mediated Warburg Effect. Biochem Biophys Res Commun 2019; 515: 85-91. https://doi.org/10.1016/j. bbrc.2019.05.111

[26] KONG X, QI J, YAN Y, CHEN L, ZHAO Y et al. Comprehensive analysis of differentially expressed profiles of lncRNAs, mRNAs, and miRNAs in laryngeal squamous cell carcinoma in order to construct a ceRNA network and identify potential biomarkers. J Cell Biochem 2019; 120: 17963-17974. https:// doi.org/10.1002/jcb.29063

[27] WANG P, WU T, ZHOU H, JIN Q, HE G et al. Long noncoding RNA NEAT1 promotes laryngeal squamous cell cancer through regulating miR-107/CDK6 pathway. J Exp Clin Cancer Res 2016; 35: 22. https://doi.org/10.1186/s13046$016-0297-Z$

[28] XU Z, XI K. LncRNA RGMB-AS1 promotes laryngeal squamous cell carcinoma cells progression via sponging miR22/NLRP3 axis. Biomed Pharmacother 2019; 118: 109222. https://doi.org/10.1016/j.biopha.2019.109222

[29] XU Q, LIU H, YU B, CHEN W, ZHAI L et al. Long noncoding RNA ZEB2-AS1 facilitates laryngeal squamous cell carcinoma progression by miR-6840-3p/PLXNB1 axis. Onco Targets Ther 2019; 12: 7337-7345. https://doi.org/10.2147/ OTT.S212749

[30] LIU L, NI J, HE X. Upregulation of the Long Noncoding RNA SNHG3 Promotes Lung Adenocarcinoma Proliferation. Dis Markers 2018; 2018: 5736716. https://doi. org/10.1155/2018/5736716

[31] XU P, LI Y, ZHANG H, LI M, ZHU H. MicroRNA-340 Mediates Metabolic Shift in Oral Squamous Cell Carcinoma by Targeting Glucose Transporter-1. J Oral Maxillofac Surg 2016; 74: 844-850. https://doi.org/10.1016/j. joms.2015.09.038

[32] GAO C, HU S. miR-506 is a YAP1-dependent tumor suppressor in laryngeal squamous cell carcinoma. Cancer Biol Ther 2019; 20: 826-836. https://doi.org/10.1080/15384047.2 018.1564569 
[33] ROSENBLUH J, WANG X, HAHN WC. Genomic insights into WNT/beta-catenin signaling. Trends Pharmacol Sci 2014; 35: 103-109. https://doi.org/10.1016/j.tips.2013.11.007

[34] SUN S, GONG C, YUAN K. LncRNA UCA1 promotes cell proliferation, invasion and migration of laryngeal squamous cell carcinoma cells by activating Wnt/beta-catenin signaling pathway. Exp Ther Med 2019; 17: 1182-1189. https://doi. org/10.3892/etm.2018.7097

[35] HE Y, DAVIES CM, HARRINGTON BS, HELLMERS L, SHENG Y et al. CDCP1 enhances Wnt signaling in colorectal cancer promoting nuclear localization of beta-catenin and E-cadherin. Oncogene 2020; 39: 219-233. https://doi. org/10.1038/s41388-019-0983-3

[36] ZHOU Q, CHEN S, LU M, LUO Y, WANG G et al. EFEMP2 suppresses epithelial-mesenchymal transition via Wnt/betacatenin signaling pathway in human bladder cancer. Int J Biol Sci 2019; 15: 2139-2155. https://doi.org/10.7150/ijbs.35541
[37] YAN Y, YAN H, WANG Q, ZHANG L, LIU Y et al. MicroRNA 10a induces glioma tumorigenesis by targeting myotubularin-related protein 3 and regulating the Wnt/beta-catenin signaling pathway. FEBS J 2019; 286: 2577-2592. https://doi.org/10.1111/febs.14824

[38] SHI S, CHEN X, LIU H, YU K, BAO Y et al. LGR5 acts as a target of miR-340-5p in the suppression of cell progression and drug resistance in breast cancer via Wnt/beta-catenin pathway. Gene 2019; 683: 47-53. https://doi.org/10.1016/j. gene.2018.10.014

[39] DENG F, PENG L, LI Z, TAN G, LIANG E et al. YAP triggers the Wnt/beta-catenin signalling pathway and promotes enterocyte self-renewal, regeneration and tumorigenesis after DSS-induced injury. Cell Death Dis 2018; 9: 153. https:// doi.org/10.1038/s41419-017-0244-8 